\title{
A RULE SET \\ TO SELECT REPRESENTATIVE NOUNS FROM A NOUN SYNONYM SET FOR A JAPANESE FISHING WEBSITE
}

\author{
Kenji Kawabata \\ Kyushu University \\ Motooka 744, Nishi-Ku, Fukuoka-Shi, 819-0395, Japan \\ te106109@s.kyushu-u.ac.jp \\ Kunihiko Kaneko \\ Kyushu University \\ Motooka 744, Nishi-Ku, Fukuoka-Shi, 819-0395, Japan \\ kaneko@ait.kyushu-u.ac.jp
}

\begin{abstract}
Japanese documents have noun synonyms. These use kanji notation, hiragana notation, and katakana notation for words. Sometimes words have alternate kanji expressions: alternate names for an object, different suffixes for kanji, etc. This is why noun synonym sets are formed for Japanese nouns. Thesauruses and dictionaries can be used to select a representative expression from a noun synonym set. However, these references do not consider the type of document. Representative nouns are often different depending on the type of articles. For example, in articles in newspapers, kanji is preferred. In contrast, in articles in encyclopedias, katakana is preferred. The problem is to form a rule set to select a representative noun from a noun synonym set, and the rule set must consider the type of document. We propose a rule set arranged for the WEB Fish Encyclopedia (in Japanese, Sakanazukan). We introduce a keyword category in the rule set to increase the correctness of the selected representative noun. As a result, most of the representative expressions were selected appropriately from noun synonyms. We expressed these noun synonyms as feature vectors. By using three numerical values and four Boolean values, all noun synonyms were expressed.
\end{abstract}

Keywords: Noun Synonym, Japanese Syntax Analysis, Keyword Dictionary 


\section{INTRODUCTION}

Selecting representative nouns from noun synonym sets is an important issue in the document database research area. We accomplish this by using thesauruses and dictionaries. However, we assume that existing dictionaries, such as the Japanese dictionary Kojien ${ }^{1}$ or Wikipedia ${ }^{2}$, cannot cover all nouns, especially nouns specific to a particular field. Therefore, we need to create a rule set to select the representative nouns from noun synonym sets. Representative nouns are often different depending on the types of articles. For example, in Japanese documents, nouns are represented using kanji notation, hiragana notation, and katakana notation. In articles in newspapers, kanji is preferred. In contrast, in articles in encyclopedias, katakana is preferred. Furthermore, for Japanese documents in general, the problem is complicated by the fact that sometimes words have alternate kanji expressions: alternate names for an object, different suffixes for kanji, etc. Until now, a rule set to select a representative noun from a noun synonym set for Japanese documents has not been studied by any researchers as far as we know. We analyze the orthographical variants of the nouns in the WEB Fish Encyclopedia ${ }^{3}$, and we construct a customized rule set that works well for the WEB Fish Encyclopedia. The organization of this paper is as follows. Section 2 briefly explains a method to extract Japanese nouns from Japanese documents. The details of the customized rule set are explained in Section 3. Section 4 explains examples of usages of the representative nouns of Japanese documents.

\section{EXTRACTION OF NOUNS}

Japanese has three different types of characters. One is hiragana (cursive Japanese syllabary), another is katakana (angular Japanese syllabary), and the other is kanji (Chinese characters). For example, if we want to write "moss" in Japanese, the hiragana expression is "こけ", the katakana expression is “コケ”, and the kanji expression is “苔”. The exact meaning can be expressed by using kanji, and the sound of the word can be expressed by hiragana and katakana. 


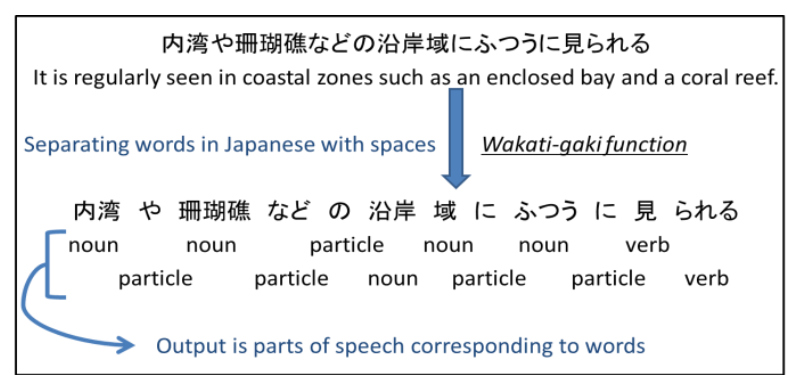

Figure 1.The WEB fish encyclopedia was separated into words using $\mathrm{MeCab}$

We installed and operated the MeCabsoftware to extract nouns fromthe WEB Fish Encyclopedia(in Japanese, Sakanazukan) ${ }^{3}$. The WEB Fish Encyclopedia contains 4,668 files, contributed by many anonymous authors. Therefore, we cannot avoid the occurrence of orthographical variants caused by the method of contribution. The WEB Fish Encyclopedia was separated into words using MeCab. We obtained 1,365,149 words, including 9,545 nouns. Each noun is either kanji, hiragana, katakana, or a mixture.

Table 1. List of orthographical variants

\begin{tabular}{|c|c|c|c|}
\hline English & Japanese & Orthographical variants & \\
\hline Coudal fin & 尾鯺 & 尾鯺, 尾びれ & \multirow{6}{*}{ Fully kanji or partly } \\
\hline Dorsal fin & 背鯺 & 背鯺, 背びれ & \\
\hline Mustache & 口髭 & ロ髭, ロひげ & \\
\hline All over the world & 世界中 & 世界中, 世界じゅう & \\
\hline Boiled food & 煮付け & 煮付け, 煮つけ & \\
\hline Hatching & 卵孚化 & 卵孚化, 心化 & \\
\hline Snakehead & ライギョ & ライギョ, 雷魚 & Kanji or katakana \\
\hline Wedge & くさび & くさび, クサビ & \multirow{5}{*}{ Hiragana or katakana } \\
\hline Fold & ひだ & ひだ，ヒダ & \\
\hline Puffer fish & フグ & フグ, ふぐ & \\
\hline Kusaya & くさや & くさや, クサヤ & \\
\hline Oyster & カキ & カキ, かき & \\
\hline Month & 力月 & 力月, 力月 & Different counter suffix \\
\hline Shop counter & 売場 & 売場, 売り場 & \multirow{3}{*}{$\begin{array}{l}\text { Different declensional } \\
\text { hiragana ending }\end{array}$} \\
\hline Treatment & 取扱い & 取扱い, 取り扱い & \\
\hline Fish paste & 練製品 & 練製品, 練り製品 & \\
\hline Yaeyama Islands & 八重山諸島 & $\begin{array}{c}\text { 八重山諸島, 八重 } \\
\text { 山列島 }\end{array}$ & \multirow{2}{*}{ Alternate names } \\
\hline Radial & 放射状 & 放射状, 放射線状 & \\
\hline
\end{tabular}


Table 1. List of orthographical variants (Cont.)

\begin{tabular}{|c|c|c|c|}
\hline English & Japanese & $\begin{array}{c}\text { Orthographical } \\
\text { variants }\end{array}$ & \\
\hline First & 一番 & 一番, いちばん & \multirow{18}{*}{ Kanji or not } \\
\hline Part & 一部 & 一部，いちぶ & \\
\hline Now & 今 & 今, いま & \\
\hline Place & 場所 & 場所, ばしよ & \\
\hline Human & 人 & 人, ヒト, ひと & \\
\hline Territory & 縄張り & 縄張り，なわばり & \\
\hline Almost & 殆ど & 殆ど, ほとんど & \\
\hline All & 全て & 全て, すべて & \\
\hline Loose & 緩やか & 緩やか，ゆるやか & \\
\hline Mortar & すり鉢 & すり鉢，すりばち & \\
\hline Moss & 苔 & 苔, コケ, こけ & \\
\hline Shape & 形 & 形，かたち & \\
\hline Varied & 様々 & 様々，さまざま & \\
\hline Two & 二つ & 二つ, ふたつ & \\
\hline Barbel & 髹 & 髭，䰅，ヒゲ，ひげ & \\
\hline Other & その他 & その他, そのた & \\
\hline Roundness & 丸み & 丸み，まるみ & \\
\hline Fin & 鯺 & 鯺，ヒレ & \\
\hline Category & カテゴリー & カテゴリー, カテゴ & \multirow{9}{*}{$\begin{array}{c}\text { Existence of a long } \\
\text { vowel }\end{array}$} \\
\hline Superior & スペリオル & $\begin{array}{c}\text { スペリオル, スペリ } \\
\text { オール }\end{array}$ & \\
\hline Pair & ペア & ペア, ペアー & \\
\hline Orange raffia & オレンジラフィ & $\begin{array}{l}\text { オレンジラフィ, } \\
\text { オレンジラフィー }\end{array}$ & \\
\hline Manitoba & マニトバ & マニトバ, マニトー & \\
\hline Laccadive & ラカディヴ & $\begin{array}{c}\text { ラカディヴ, ラカデ } \\
\text { イーヴ }\end{array}$ & \\
\hline Bermuda & バミューダ & $\begin{array}{c}\text { バミューダ，バーミ } \\
\text { ューダ }\end{array}$ & \\
\hline $\begin{array}{l}\text { New South } \\
\text { Wales }\end{array}$ & $\begin{array}{c}\text { ニューサウスウェー } \\
\text { ルズ }\end{array}$ & $\begin{array}{c}\text { ニューサウスウェー } \\
\text { ルズ, } \\
\text { ニューサウスウェル } \\
\text { ズ }\end{array}$ & \\
\hline Chao Phraya & チャオプラヤ & $\begin{array}{c}\text { チャオプラヤ, チャ } \\
\text { オプラヤー }\end{array}$ & \\
\hline
\end{tabular}


Table 1. List of orthographical variants (Cont.)

\begin{tabular}{|c|c|c|c|}
\hline English & Japanese & $\begin{array}{c}\text { Orthographical } \\
\text { variants }\end{array}$ & \\
\hline $\begin{array}{l}\text { Papua New } \\
\text { Guinea }\end{array}$ & $\begin{array}{c}\text { バハカリフォルニ } \\
\text { ア } \\
\text { パプアニューギニ } \\
\text { ア }\end{array}$ & $\begin{array}{c}\text { バハカリフォルニア, } \\
\text { バハ・カリフォルニ } \\
\text { ア } \\
\text { パプアニューギニア, } \\
\text { パプア・ニューギニ } \\
\text { ア }\end{array}$ & $\begin{array}{l}\text { Existence of a bullet } \\
\text { point }\end{array}$ \\
\hline $\begin{array}{l}\text { Suffix use: when } \\
\text { Spanish } \\
\text { mackerel } \\
\end{array}$ & $\begin{array}{l}\text { ころ } \\
\text { サワラ } \\
\end{array}$ & $\begin{array}{c}\text { ころ, ごろ } \\
\text { サワラ, ザワラ } \\
\end{array}$ & $\begin{array}{l}\text { Difference caused by } \\
\text { taking } \\
\text { along to other nouns }\end{array}$ \\
\hline Leptocephalus & レプトセファルス & $\begin{array}{c}\text { カムチャツカ, カム } \\
\text { チャッカ } \\
\text { レプトセファルス, } \\
\text { レプトケファルス, } \\
\text { レプトケパルス }\end{array}$ & \multirow{13}{*}{$\begin{array}{l}\text { Different katakana } \\
\text { expression }\end{array}$} \\
\hline Timor & チモール & $\begin{array}{c}\text { チモール, ティモー } \\
\text { ル }\end{array}$ & \\
\hline Philipin & フィリピン & $\begin{array}{c}\text { フィリピン, フィリ } \\
\text { ンピン }\end{array}$ & \\
\hline Diver & ダイバー & ダイバー, ダイヴァ & \\
\hline Kermadec & ケルマデック & $\begin{array}{c}\text { ケルマデック, ケル } \\
\text { マディック }\end{array}$ & \\
\hline Lord Howe & ロードハウ & $\begin{array}{c}\text { ロードハウ, ロード } \\
\text { ホウ }\end{array}$ & \\
\hline Tuamotu & トゥアモトゥ & $\begin{array}{c}\text { トゥアモトゥ, ツア } \\
\text { モツ }\end{array}$ & \\
\hline Maldive & モルディブ & $\begin{array}{c}\text { モルディブ, モルジ } \\
\text { ブ, } \\
\text { モルディヴ }\end{array}$ & \\
\hline Loyalty & ロイヤルティ & $\begin{array}{c}\text { ロイヤルティ, ロヤ } \\
\text { ルティ } \\
\text { ソサエティ, ソサ }\end{array}$ & \\
\hline Society & ソサイエティ & $\begin{array}{l}\text { エティ, } \\
\text { ソサイティ }\end{array}$ & \\
\hline Pitcairn & ピトケアン & $\begin{array}{c}\text { ピトケアン, ピトカ } \\
\text { ーン }\end{array}$ & \\
\hline Nova Scotia & ノバスコシア & $\begin{array}{c}\text { ノバスコシア, ノヴ } \\
\text { アスコシア }\end{array}$ & \\
\hline Marquesas & マルケサス & $\begin{array}{c}\text { マルケサス, マーケ } \\
\text { サス }\end{array}$ & \\
\hline
\end{tabular}


Table 1. List of orthographical variants (Cont.)

\begin{tabular}{|c|c|c|c|}
\hline English & Japanese & $\begin{array}{c}\text { Orthographical } \\
\text { variants }\end{array}$ & \\
\hline Monterey & モンタレイ & $\begin{array}{c}\text { モンタレイ, モン } \\
\text { タレー }\end{array}$ & \\
\hline Cobitis & スジシマドジョウ & $\begin{array}{l}\text { スジシマドジョウ, } \\
\text { スジシマドショウ }\end{array}$ & \\
\hline Sardinella melanura & オグロイワシ & $\begin{array}{c}\text { オグロイワシ，オ } \\
\text { グロオワシ }\end{array}$ & Different \\
\hline Tetrodotoxin & テトロドトキシン & $\begin{array}{l}\text { テトロドトキシン, } \\
\text { テトロドドキシン } \\
\text { サスカチュワン, }\end{array}$ & $\begin{array}{l}\text { katakana } \\
\text { expression }\end{array}$ \\
\hline Saskatchewan & サスカチュワン & $\begin{array}{c}\text { サスカッチュュワ } \\
\text { ン }\end{array}$ & \\
\hline Georgia & ジョージア & $\begin{array}{l}\text { ジョージア, } \\
\text { ジュージア }\end{array}$ & \\
\hline Great Barrier Reef & グレートバリアリーフ & $\begin{array}{c}\text { グレートバリアリ } \\
\text {-フ, } \\
\text { グレイトバリアリ } \\
\text {-フ, } \\
\text { グレートバリアー } \\
\text { リーフ } \\
\text { ニュージーランド, } \\
\text { ニュージランド, } \\
\text { ニュージーーラン } \\
\text { ド }\end{array}$ & $\begin{array}{c}\text { Different } \\
\text { katakana } \\
\text { expression } \\
\text { and a long vowel }\end{array}$ \\
\hline
\end{tabular}

\section{SELECTING KEYWORDS}

\subsection{Orthographical Variants}

As stated above, the noun list contains orthographical variants of noun synonyms depending on the content and the contributors. When a Japanese text describes English names and the names of other countries in katakana, it produces several other descriptions. Differences are also found if the text is in kanji. We found 72 noun synonym sets from the WEB Fish Encyclopedia, and we selected a representative noun fromeach set manually. We use each noun as the ground truth. Then, we use the term "orthographical variants" to refer to nouns other than the representative nouns in the noun synonym sets. In all, we categorized the 72 noun synonym sets into 11 types according to their differences. Table 2 shows the representative nouns, orthographical variants, and categorized results. As we expected, most of the differences were caused by kanji and katakana expressions. Almost the same number of occurrences, such as "Kamchatka" 
(a peninsula in Russia), appeared in the files. In Japanese “カムチャツカ” appeared 7 times and “カムチャッカ” appeared 9 times. Some words such as "Great Barrier Reef" were divided into a majority notation and a minority. “グレートバリアリーフ”, in Japanese, appeared 18 times and “グレイトバリアリーフ” appeared once.

Some cases were obviously caused by an error. Our goal is to form a rule set to select representative nouns automatically from the synonym sets. We formed six rules, as shown in the following bulleted list. In the rules, each noun has attributes. These are Is_general_term $(x), I s \_p r o p e r \_n o u n(x)$, Is_katakana $(x), \quad I s \_c o m m o n \_n o u n(x), \quad I s \_k a n j i(x), \quad I s \_K o j i e n(x)$, Is_most_frequent $(x), \quad$ Is_Wikipedia_keyword $(x), \quad$ and Hit_Google_search_the_most $(x)$. We utilized the keyword entries of the Japanese dictionaryKojien fourth edition, Wikipedia, and the Google search engine.

$$
\begin{aligned}
& \text { Input_words }\left\{\exists x \mid x \in I s \_g e n e r a l \_t e r m(x) \text { or (Is_proper_noun( } x\right) \text { and } \\
& \text { Is_katakana }(x))\} \Rightarrow \text { Is_representative_notation }(x) \\
& \text { Input_words }\left\{\exists x \mid x \in I s_{-} \text {common_noun }(x) \text { and Is_kanji }(x)\right\} \\
& \Rightarrow I s \_r e p r e s e n t a t i v e \_n o t a t i o n(x) \\
& \text { Input_words }\left\{\exists x \mid x \in I s \_K o j i e n \_k e y w o r d(x)\right\} \\
& \Rightarrow I s \_r e p r e s e n t a t i v e \_n o t a t i o n(x) \\
& \text { Input_words }\left\{\exists x \mid x \in I s \_ \text {most_frequent }(x)\right\} \\
& \Rightarrow I s \_r e p r e s e n t a t i v e \_n o t a t i o n(x) \\
& \text { Input_words }\{\exists x \mid x \in I \text { Is_Wikipedia_keyword }(x)\} \\
& \Rightarrow I s \_r e p r e s e n t a t i v e \_n o t a t i o n(x) \\
& \text { Input_words }\{\exists x \mid x \in \text { Hit_Google_search_the_most }(x)\} \\
& \Rightarrow I s \_r e p r e s e n t a t i v e \_n o t a t i o n(x)
\end{aligned}
$$

We created a flowchart to select representative nouns by using the above rules. The flowchart, arranged for Sakanazukan, is shown in Fig. 2. A single representative can be selected by following this flowchart. For the general terms of fish and overseas place names in the WEB Fish Encyclopedia, the representative noun should bekatakana, and for the domestic place names and common nouns, the representative noun should be kanji. We used those rules in the flowchart. 


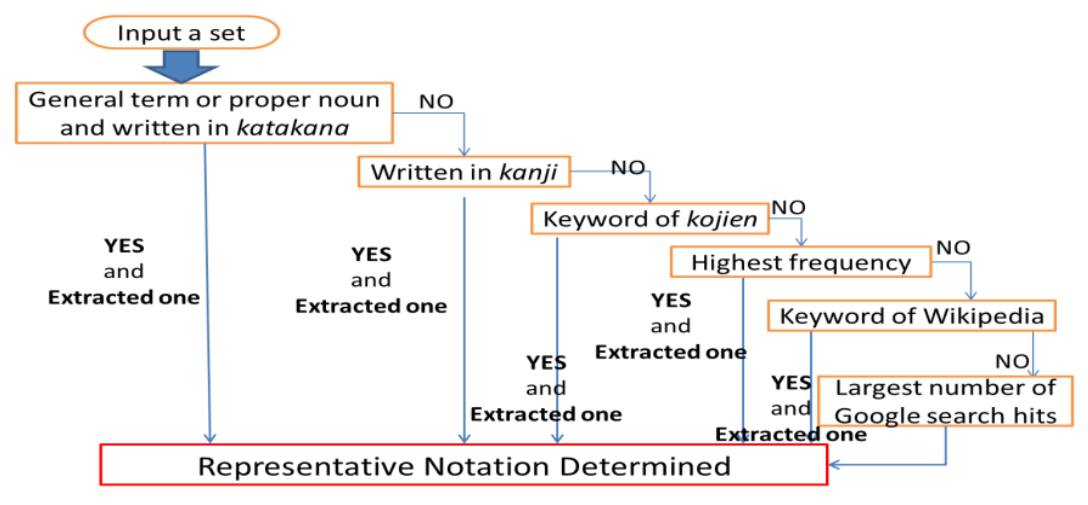

Figure 2. Flowchart of keyword selection

We also represented the noun attributes as noun feature vectors, as shown in Table 2. We selected 70 correct representative notations from the 72 noun synonym sets, and so the correctness is 70/72.

Table 2. Feature vectors of the nouns

\begin{tabular}{|c|c|c|c|c|c|c|c|}
\hline Word (in Japanese) & Katakana & $\begin{array}{c}\text { Number of } \\
\text { kanji }\end{array}$ & Hiragana & Kojien & Wikipedia & Frequency & Google Hit \\
\hline 一番 & $\mathrm{F}$ & 2 & $\mathrm{~F}$ & $\mathrm{~T}$ & $\mathrm{~F}$ & 2 & 616000000 \\
\hline いちばん & $\mathrm{F}$ & 0 & $\mathrm{~T}$ & $\mathrm{~T}$ & $\mathrm{~F}$ & 2 & 34900000 \\
\hline 一部 & $\mathrm{F}$ & 2 & $\mathrm{~F}$ & $\mathrm{~T}$ & $\mathrm{~T}$ & 50 & 526000000 \\
\hline いちぶ & $\mathrm{F}$ & 0 & $\mathrm{~T}$ & $\mathrm{~T}$ & $\mathrm{~F}$ & 1 & 270000 \\
\hline 今 & $\mathrm{F}$ & 1 & $\mathrm{~F}$ & $\mathrm{~T}$ & $\mathrm{~T}$ & 9 & 2310000000 \\
\hline いま & $\mathrm{F}$ & 0 & $\mathrm{~T}$ & $\mathrm{~T}$ & $\mathrm{~F}$ & 10 & 480000000 \\
\hline 売場 & $\mathrm{F}$ & 2 & $\mathrm{~F}$ & $\mathrm{~T}$ & $\mathrm{~F}$ & 3 & 8920000 \\
\hline 売り場 & $\mathrm{F}$ & 2 & $\mathrm{~T}$ & $\mathrm{~F}$ & $\mathrm{~F}$ & 1 & 21800000 \\
\hline オグロイワシ & $\mathrm{T}$ & 0 & $\mathrm{~F}$ & $\mathrm{~F}$ & $\mathrm{~F}$ & 1 & 89 \\
\hline オグロオワシ & $\mathrm{T}$ & 0 & $\mathrm{~F}$ & $\mathrm{~F}$ & $\mathrm{~F}$ & 2 & 1 \\
\hline 尾鰭 & $\mathrm{F}$ & 2 & $\mathrm{~F}$ & $\mathrm{~T}$ & $\mathrm{~T}$ & 1071 & 436000 \\
\hline 尾びれ & $\mathrm{F}$ & 1 & $\mathrm{~T}$ & $\mathrm{~F}$ & $\mathrm{~T}$ & 5 & 485000 \\
\hline オレンジラフィ & $\mathrm{T}$ & 0 & $\mathrm{~F}$ & $\mathrm{~F}$ & $\mathrm{~F}$ & 2 & 1140 \\
\hline オレンジラフィー & $\mathrm{T}$ & 0 & $\mathrm{~F}$ & $\mathrm{~F}$ & $\mathrm{~F}$ & 1 & 16100 \\
\hline カキ & $\mathrm{T}$ & 0 & $\mathrm{~F}$ & $\mathrm{~F}$ & $\mathrm{~T}$ & 5 & 13500000 \\
\hline かき & $\mathrm{F}$ & 0 & $\mathrm{~T}$ & $\mathrm{~T}$ & $\mathrm{~T}$ & 1 & 34000000 \\
\hline カ月 & $\mathrm{F}$ & 1 & $\mathrm{~F}$ & $\mathrm{~F}$ & $\mathrm{~T}$ & 5 & 54700000 \\
\hline カ月 & $\mathrm{T}$ & 1 & $\mathrm{~F}$ & $\mathrm{~F}$ & $\mathrm{~T}$ & 1 & 58300000 \\
\hline 形 & $\mathrm{F}$ & 1 & $\mathrm{~F}$ & $\mathrm{~T}$ & $\mathrm{~T}$ & 222 & 706000000 \\
\hline かたち & $\mathrm{F}$ & 0 & $\mathrm{~T}$ & $\mathrm{~T}$ & $\mathrm{~F}$ & 2 & 15000000 \\
\hline カテゴリー & $\mathrm{T}$ & 0 & $\mathrm{~F}$ & $\mathrm{~T}$ & $\mathrm{~T}$ & 2 & 1180000000 \\
\hline カテゴリ & $\mathrm{T}$ & 0 & $\mathrm{~F}$ & $\mathrm{~F}$ & $\mathrm{~T}$ & 1 & 1250000000 \\
\hline カムチャツカ & $\mathrm{T}$ & 0 & $\mathrm{~F}$ & $\mathrm{~T}$ & $\mathrm{~T}$ & 7 & 222000 \\
\hline カムチャッカ & $\mathrm{T}$ & 0 & $\mathrm{~F}$ & $\mathrm{~F}$ & $\mathrm{~T}$ & 9 & 307000 \\
\hline くさや & F & 0 & $\mathrm{~T}$ & $\mathrm{~T}$ & $\mathrm{~T}$ & 1 & 1420000 \\
\hline クサヤ & $\mathrm{T}$ & 0 & $\mathrm{~F}$ & $\mathrm{~F}$ & $\mathrm{~T}$ & 3 & 125000 \\
\hline くさび & $\mathrm{F}$ & 0 & $\mathrm{~T}$ & $\mathrm{~T}$ & $\mathrm{~T}$ & 4 & 948000 \\
\hline クサビ & $\mathrm{T}$ & 0 & $\mathrm{~F}$ & $\mathrm{~F}$ & $\mathrm{~T}$ & 5 & 700000 \\
\hline 口髭 & $\mathrm{F}$ & 2 & $\mathrm{~F}$ & $\mathrm{~T}$ & $\mathrm{~T}$ & 6 & 441000 \\
\hline
\end{tabular}


Table 2. Feature vectors of the nouns (Cont.)

\begin{tabular}{|c|c|c|c|c|c|c|c|}
\hline $\begin{array}{c}\text { Word } \\
\text { (in Japanese) }\end{array}$ & Katakana & $\begin{array}{c}\text { Number of } \\
\text { kanji }\end{array}$ & Hiragana & Kojien & Wikipedia & Frequency & Google Hit \\
\hline ロひげ & $\mathrm{F}$ & 1 & $\mathrm{~T}$ & $\mathrm{~F}$ & $\mathrm{~T}$ & 55 & 374000 \\
\hline $\begin{array}{l}\text { グレートバリア } \\
\text { リーフ }\end{array}$ & $\mathrm{T}$ & 0 & $\mathrm{~F}$ & $\mathrm{~F}$ & $\mathrm{~T}$ & 18 & 1410000 \\
\hline $\begin{array}{l}\text { グレイトバリア } \\
\text { リーフ }\end{array}$ & $\mathrm{T}$ & 0 & $\mathrm{~F}$ & $\mathrm{~F}$ & $\mathrm{~F}$ & 1 & 13800 \\
\hline $\begin{array}{l}\text { グレートバリア } \\
\text { ーリーフ }\end{array}$ & $\mathrm{T}$ & 0 & $\mathrm{~F}$ & $\mathrm{~F}$ & $\mathrm{~T}$ & 1 & 19100 \\
\hline ケルマデック & $\mathrm{T}$ & 0 & $\mathrm{~F}$ & $\mathrm{~F}$ & $\mathrm{~T}$ & 3 & 12100 \\
\hline ケルマディック & $\mathrm{T}$ & 0 & $\mathrm{~F}$ & $\mathrm{~F}$ & $\mathrm{~T}$ & 1 & 44900 \\
\hline 苔 & $\mathrm{F}$ & 1 & $\mathrm{~F}$ & $\mathrm{~T}$ & $\mathrm{~T}$ & 1 & 24200000 \\
\hline コケ & $\mathrm{T}$ & 0 & $\mathrm{~F}$ & $\mathrm{~F}$ & $\mathrm{~T}$ & 1 & 11600000 \\
\hline こけ & $\mathrm{F}$ & 0 & $\mathrm{~T}$ & $\mathrm{~T}$ & $\mathrm{~T}$ & 19 & 8730000 \\
\hline ころ & $\mathrm{F}$ & 0 & $\mathrm{~T}$ & $\mathrm{~T}$ & $\mathrm{~F}$ & 1 & 103000000 \\
\hline ごろ & $\mathrm{F}$ & 0 & $\mathrm{~T}$ & $\mathrm{~F}$ & $\mathrm{~F}$ & 2 & 66900000 \\
\hline サスカチュワン & $\mathrm{T}$ & 0 & $\mathrm{~F}$ & $\mathrm{~F}$ & $\mathrm{~T}$ & 1 & 207000 \\
\hline $\begin{array}{l}\text { サスカッチュュ } \\
\text { ワン }\end{array}$ & $\mathrm{T}$ & 0 & $\mathrm{~F}$ & $\mathrm{~F}$ & $\mathrm{~F}$ & 1 & 2 \\
\hline 様々 & $\mathrm{F}$ & 2 & $\mathrm{~F}$ & $\mathrm{~F}$ & $\mathrm{~F}$ & 14 & 193000000 \\
\hline さまざま & $\mathrm{F}$ & 0 & $\mathrm{~T}$ & $\mathrm{~T}$ & $\mathrm{~F}$ & 8 & 107000000 \\
\hline サワラ & $\mathrm{T}$ & 0 & $\mathrm{~F}$ & $\mathrm{~F}$ & $\mathrm{~T}$ & 10 & 1570000 \\
\hline ザワラ & $\mathrm{T}$ & 0 & $\mathrm{~F}$ & $\mathrm{~F}$ & $\mathrm{~F}$ & 1 & 6130 \\
\hline ジョージア & $\mathrm{T}$ & 0 & $\mathrm{~F}$ & $\mathrm{~T}$ & $\mathrm{~T}$ & 4 & 5600000 \\
\hline ジュージア & $\mathrm{T}$ & 0 & $\mathrm{~F}$ & $\mathrm{~F}$ & $\mathrm{~F}$ & 1 & 6810 \\
\hline $\begin{array}{l}\text { スジシマドジョ } \\
\text { ウ }\end{array}$ & $\mathrm{T}$ & 0 & $\mathrm{~F}$ & $\mathrm{~F}$ & $\mathrm{~F}$ & 6 & 108000 \\
\hline $\begin{array}{l}\text { スジシマドショ } \\
\text { ウ }\end{array}$ & $\mathrm{T}$ & 0 & $\mathrm{~F}$ & $\mathrm{~F}$ & $\mathrm{~F}$ & 1 & 278 \\
\hline 全て & $\mathrm{F}$ & 1 & $\mathrm{~T}$ & $\mathrm{~T}$ & $\mathrm{~T}$ & 14 & 617000000 \\
\hline すべて & $\mathrm{F}$ & 0 & $\mathrm{~T}$ & $\mathrm{~T}$ & $\mathrm{~F}$ & 7 & $\begin{array}{r}244000000 \\
0\end{array}$ \\
\hline スペリオル & $\mathrm{T}$ & 0 & $\mathrm{~F}$ & $\mathrm{~T}$ & $\mathrm{~T}$ & 1 & 618000 \\
\hline スペリオール & $\mathrm{T}$ & 0 & $\mathrm{~F}$ & $\mathrm{~F}$ & $\mathrm{~F}$ & 1 & 1120000 \\
\hline すり鉢 & $\mathrm{F}$ & 1 & $\mathrm{~T}$ & $\mathrm{~F}$ & $\mathrm{~T}$ & 5 & 1760000 \\
\hline すりばち & $\mathrm{F}$ & 0 & $\mathrm{~T}$ & $\mathrm{~T}$ & $\mathrm{~T}$ & 2 & 133000 \\
\hline 世界中 & $\mathrm{F}$ & 3 & $\mathrm{~F}$ & $\mathrm{~F}$ & $\mathrm{~F}$ & 60 & 230000000 \\
\hline 世界じゅう & F & 2 & $\mathrm{~T}$ & $\mathrm{~F}$ & $\mathrm{~F}$ & 2 & 333000 \\
\hline 背鯺 & $\mathrm{F}$ & 2 & $\mathrm{~F}$ & $\mathrm{~T}$ & $\mathrm{~T}$ & 2189 & 372000 \\
\hline 背びれ & F & 1 & $\mathrm{~T}$ & F & $\mathrm{T}$ & 1 & 522000 \\
\hline ソサイエティ & $\mathrm{T}$ & 0 & $\mathrm{~F}$ & $\mathrm{~F}$ & $\mathrm{~F}$ & 3 & 627000 \\
\hline ソサエティ & $\mathrm{T}$ & 0 & $\mathrm{~F}$ & $\mathrm{~F}$ & $\mathrm{~F}$ & 1 & 1860000 \\
\hline ソサイティ & $\mathrm{T}$ & 0 & $\mathrm{~F}$ & $\mathrm{~F}$ & $\mathrm{~F}$ & 1 & 12300 \\
\hline その他 & F & 1 & $\mathrm{~T}$ & $\mathrm{~F}$ & $\mathrm{~T}$ & 1143 & $\begin{array}{r}167000000 \\
0\end{array}$ \\
\hline そのほか & $\mathrm{F}$ & 0 & $\mathrm{~T}$ & $\mathrm{~F}$ & $\mathrm{~F}$ & 1 & 45800000 \\
\hline ダイバー & $\mathrm{T}$ & 0 & $\mathrm{~F}$ & $\mathrm{~T}$ & $\mathrm{~T}$ & 14 & 9390000 \\
\hline ダイヴァー & $\mathrm{T}$ & 0 & $\mathrm{~F}$ & $\mathrm{~F}$ & F & 4 & 78800 \\
\hline チモール & $\mathrm{T}$ & 0 & $\mathrm{~F}$ & $\mathrm{~T}$ & $\mathrm{~T}$ & 6 & 272000 \\
\hline ティモール & $\mathrm{T}$ & 0 & $\mathrm{~F}$ & $\mathrm{~F}$ & $\mathrm{~T}$ & 2 & 3370000 \\
\hline チャオプラヤ & $\mathrm{T}$ & 0 & $\mathrm{~F}$ & $\mathrm{~T}$ & $\mathrm{~F}$ & 1 & 925000 \\
\hline チャオプラヤー & $\mathrm{T}$ & 0 & $\mathrm{~F}$ & $\mathrm{~F}$ & $\mathrm{~T}$ & 1 & 490000 \\
\hline $\begin{array}{l}\text { テトロドトキシ } \\
\text { ン }\end{array}$ & $\mathrm{T}$ & 0 & F & $\mathrm{T}$ & $\mathrm{T}$ & 48 & 92700 \\
\hline $\begin{array}{l}\text { テトロドドキシ } \\
\text { ン }\end{array}$ & $\mathrm{T}$ & 0 & $\mathrm{~F}$ & $\mathrm{~F}$ & $\mathrm{~F}$ & 1 & 2850 \\
\hline
\end{tabular}


Table 2. Feature vectors of the nouns (Cont.)

\begin{tabular}{|c|c|c|c|c|c|c|c|}
\hline $\begin{array}{c}\text { Word } \\
\text { (in Japanese) }\end{array}$ & Katakana & $\begin{array}{l}\text { Number } \\
\text { of kanji }\end{array}$ & Hiragana & Kojien & Wikipedia & Frequency & Google Hit \\
\hline トゥアモトゥ & $\mathrm{T}$ & 0 & F & F & $\mathrm{F}$ & 3 & 6410 \\
\hline ツアモッ & $\mathrm{T}$ & 0 & $\mathrm{~F}$ & $\mathrm{~F}$ & $\mathrm{~F}$ & 1 & 25800 \\
\hline 取扱い & $\mathrm{F}$ & 2 & $\mathrm{~T}$ & $\mathrm{~T}$ & $\mathrm{~F}$ & 1 & 66200000 \\
\hline 取り扱い & $\mathrm{F}$ & 2 & $\mathrm{~T}$ & $\mathrm{~F}$ & $\mathrm{~F}$ & 1 & 174000000 \\
\hline 縄張り & $\mathrm{F}$ & 2 & $\mathrm{~T}$ & $\mathrm{~F}$ & $\mathrm{~T}$ & 21 & 2490000 \\
\hline $\begin{array}{l}\text { ニューサウスウェ } \\
\text { ールズ }\end{array}$ & $\mathrm{T}$ & 0 & $\mathrm{~F}$ & $\mathrm{~T}$ & $\mathrm{~T}$ & 4 & 939000 \\
\hline $\begin{array}{l}\text { ニューサウスウェ } \\
\text { ルズ }\end{array}$ & $\mathrm{T}$ & 0 & $\mathrm{~F}$ & $\mathrm{~F}$ & $\mathrm{~F}$ & 7 & 11500 \\
\hline ニュージーランド & $\mathrm{T}$ & 0 & $\mathrm{~F}$ & $\mathrm{~T}$ & $\mathrm{~T}$ & 108 & 24100000 \\
\hline ニュージランド & $\mathrm{T}$ & 0 & $\mathrm{~F}$ & $\mathrm{~F}$ & $\mathrm{~F}$ & 4 & 529000 \\
\hline $\begin{array}{l}\text { ニュージーーラン } \\
\text { ド }\end{array}$ & $\mathrm{T}$ & 0 & $\mathrm{~F}$ & $\mathrm{~F}$ & $\mathrm{~F}$ & 1 & 5440 \\
\hline 煮付け & $\mathrm{F}$ & 2 & $\mathrm{~T}$ & $\mathrm{~F}$ & $\mathrm{~T}$ & 3 & 5020000 \\
\hline 煮つけ & $\mathrm{F}$ & 1 & $\mathrm{~T}$ & $\mathrm{~F}$ & $\mathrm{~F}$ & 4 & 1210000 \\
\hline 練製品 & $\mathrm{F}$ & 3 & F & $\mathrm{T}$ & $\mathrm{F}$ & 31 & 285000 \\
\hline 練り製品 & $\mathrm{F}$ & 3 & $\mathrm{~T}$ & $\mathrm{~F}$ & $\mathrm{~T}$ & 4 & 319000 \\
\hline ハバスコシア & $\mathrm{T}$ & 0 & $\mathrm{~F}$ & $\mathrm{~F}$ & $\mathrm{~T}$ & 5 & 253000 \\
\hline ノヴァスコシア & $\mathrm{T}$ & 0 & $\mathrm{~F}$ & $\mathrm{~F}$ & $\mathrm{~T}$ & 2 & 26200 \\
\hline 場所 & $\mathrm{F}$ & 2 & $\mathrm{~F}$ & $\mathrm{~T}$ & $\mathrm{~T}$ & 175 & 583000000 \\
\hline ばしょ & $\mathrm{F}$ & 0 & $\mathrm{~T}$ & $\mathrm{~T}$ & $\mathrm{~F}$ & 1 & 2520000 \\
\hline $\begin{array}{l}\text { バハカリフォルニ } \\
\text { ア }\end{array}$ & $\mathrm{T}$ & 0 & $\mathrm{~F}$ & $\mathrm{~F}$ & $\mathrm{~T}$ & 29 & 342000 \\
\hline $\begin{array}{l}\text { バハ·カリフォル } \\
\text { ニア }\end{array}$ & $\mathrm{T}$ & 0 & $\mathrm{~F}$ & $\mathrm{~F}$ & $\mathrm{~T}$ & 4 & 342000 \\
\hline $\begin{array}{l}\text { パプアニューギニ } \\
\text { ア }\end{array}$ & $\mathrm{T}$ & 0 & $\mathrm{~F}$ & $\mathrm{~F}$ & $\mathrm{~T}$ & 33 & 5000000 \\
\hline $\begin{array}{l}\text { パプア·ニューギ } \\
\text { ニア }\end{array}$ & $\mathrm{T}$ & 0 & $\mathrm{~F}$ & $\mathrm{~F}$ & $\mathrm{~T}$ & 2 & 5010000 \\
\hline バミューダ & $\mathrm{T}$ & 0 & $\mathrm{~F}$ & F & $\mathrm{T}$ & 26 & 2840000 \\
\hline バーミューダ & $\mathrm{T}$ & 0 & $\mathrm{~F}$ & F & $\mathrm{T}$ & 2 & 237000 \\
\hline 髭 & $\mathrm{F}$ & 1 & F & $\mathrm{T}$ & $\mathrm{T}$ & 69 & 16500000 \\
\hline 鬚 & $\mathrm{F}$ & 1 & $\mathrm{~F}$ & $\mathrm{~T}$ & $\mathrm{~T}$ & 12 & 4760000 \\
\hline ヒゲ & $\mathrm{T}$ & 0 & $\mathrm{~F}$ & $\mathrm{~F}$ & $\mathrm{~T}$ & 49 & 17700000 \\
\hline ひげ & $\mathrm{F}$ & 0 & $\mathrm{~T}$ & $\mathrm{~T}$ & $\mathrm{~T}$ & 29 & 19100000 \\
\hline ひだ & $\mathrm{F}$ & 0 & $\mathrm{~T}$ & $\mathrm{~T}$ & $\mathrm{~T}$ & 2 & 6840000 \\
\hline ヒダ & $\mathrm{T}$ & 0 & $\mathrm{~F}$ & F & F & 1 & 2230000 \\
\hline 人 & F & 1 & F & $\mathrm{T}$ & $\mathrm{T}$ & 105 & $\begin{array}{r}1228000000 \\
0\end{array}$ \\
\hline 나 & $\mathrm{T}$ & 0 & $\mathrm{~F}$ & $\mathrm{~F}$ & $\mathrm{~T}$ & 2 & 34900000 \\
\hline ひと & $\mathrm{F}$ & 0 & $\mathrm{~T}$ & $\mathrm{~T}$ & $\mathrm{~T}$ & 1 & 207000000 \\
\hline ピトケアン & $\mathrm{T}$ & 0 & $\mathrm{~F}$ & F & $\mathrm{T}$ & 1 & 788000 \\
\hline ピトカーン & $\mathrm{T}$ & 0 & $\mathrm{~F}$ & $\mathrm{~F}$ & $\mathrm{~F}$ & 1 & 1630 \\
\hline 鰭 & F & 1 & F & $\mathrm{T}$ & $\mathrm{T}$ & 2705 & 2870000 \\
\hline ヒレ & $\mathrm{T}$ & 0 & $\mathrm{~F}$ & $\mathrm{~F}$ & $\mathrm{~T}$ & 21 & 6000000 \\
\hline フィリピン & $\mathrm{T}$ & 0 & $\mathrm{~F}$ & $\mathrm{~T}$ & $\mathrm{~T}$ & 438 & 28400000 \\
\hline フィリンピン & $\mathrm{T}$ & 0 & $\mathrm{~F}$ & $\mathrm{~F}$ & $\mathrm{~F}$ & 3 & 8430 \\
\hline 孵化 & F & 2 & $\mathrm{~F}$ & $\mathrm{~T}$ & $\mathrm{~T}$ & 18 & 25600000 \\
\hline 心化 & $\mathrm{F}$ & 1 & $\mathrm{~T}$ & $\mathrm{~F}$ & $\mathrm{~T}$ & 3 & 792000 \\
\hline フグ & $\mathrm{T}$ & 0 & $\mathrm{~F}$ & $\mathrm{~F}$ & $\mathrm{~T}$ & 371 & 6230000 \\
\hline ふぐ & $\mathrm{F}$ & 0 & $\mathrm{~T}$ & $\mathrm{~T}$ & $\mathrm{~T}$ & 99 & 24200000 \\
\hline ニつ & $\mathrm{F}$ & 1 & $\mathrm{~T}$ & $\mathrm{~T}$ & $\mathrm{~F}$ & 4 & 76100000 \\
\hline ふたつ & F & 0 & $\mathrm{~T}$ & $\mathrm{~T}$ & $\mathrm{~F}$ & 1 & 16200000 \\
\hline ペア & $\mathrm{T}$ & 0 & $\mathrm{~F}$ & $\mathrm{~T}$ & $\mathrm{~T}$ & 28 & 54400000 \\
\hline
\end{tabular}


Table 2. Feature vectors of the nouns (Cont.)

\begin{tabular}{|c|c|c|c|c|c|c|c|}
\hline $\begin{array}{c}\text { Word } \\
\text { (in Japanese) }\end{array}$ & Katakana & $\begin{array}{l}\text { Number } \\
\text { of kanji }\end{array}$ & Hiragana & Kojien & Wikipedia & Frequency & Google Hit \\
\hline ペアー & $\mathrm{T}$ & 0 & F & F & $\mathrm{F}$ & 1 & 1570000 \\
\hline 放射状 & $\mathrm{F}$ & 3 & $\mathrm{~F}$ & $\mathrm{~T}$ & $\mathrm{~F}$ & 12 & 2840000 \\
\hline 放射線状 & $\mathrm{F}$ & 4 & $\mathrm{~F}$ & $\mathrm{~F}$ & $\mathrm{~F}$ & 4 & 462000 \\
\hline 殆ど & F & 1 & $\mathrm{~T}$ & $\mathrm{~T}$ & $\mathrm{~F}$ & 14 & 50800000 \\
\hline ほとんど & F & 0 & $\mathrm{~T}$ & $\mathrm{~T}$ & F & 82 & 257000000 \\
\hline マニトバ & $\mathrm{T}$ & 0 & $\mathrm{~F}$ & $\mathrm{~F}$ & $\mathrm{~T}$ & 1 & 259000 \\
\hline マニトーバ & $\mathrm{T}$ & 0 & $\mathrm{~F}$ & F & $\mathrm{F}$ & 1 & 289 \\
\hline マルケサス & $\mathrm{T}$ & 0 & $\mathrm{~F}$ & $\mathrm{~T}$ & $\mathrm{~F}$ & 1 & 37100 \\
\hline マーケサス & $\mathrm{T}$ & 0 & $\mathrm{~F}$ & $\mathrm{~F}$ & $\mathrm{~F}$ & 1 & 4820 \\
\hline 丸み & F & 1 & $\mathrm{~T}$ & $\mathrm{~T}$ & F & 45 & 6170000 \\
\hline まるみ & $\mathrm{F}$ & 0 & $\mathrm{~T}$ & $\mathrm{~T}$ & $\mathrm{~F}$ & 3 & 1840000 \\
\hline モルディブ & $\mathrm{T}$ & 0 & $\mathrm{~F}$ & $\mathrm{~F}$ & $\mathrm{~T}$ & 22 & 5030000 \\
\hline モルジブ & $\mathrm{T}$ & 0 & $\mathrm{~F}$ & $\mathrm{~F}$ & $\mathrm{~F}$ & 4 & 1830000 \\
\hline モルディヴ & $\mathrm{T}$ & 0 & $\mathrm{~F}$ & $\mathrm{~F}$ & $\mathrm{~F}$ & 2 & 152000 \\
\hline モンタレイ & $\mathrm{T}$ & 0 & $\mathrm{~F}$ & $\mathrm{~F}$ & $\mathrm{~F}$ & 4 & 6420 \\
\hline モンタレー & $\mathrm{T}$ & 0 & $\mathrm{~F}$ & $\mathrm{~F}$ & $\mathrm{~T}$ & 1 & 148000 \\
\hline 八重山諸島 & $\mathrm{F}$ & 5 & F & $\mathrm{T}$ & $\mathrm{T}$ & 43 & 3670000 \\
\hline 八重山列島 & F & 5 & $\mathrm{~F}$ & F & $\mathrm{T}$ & 6 & 183000 \\
\hline 緩やか & $\mathrm{F}$ & 1 & $\mathrm{~T}$ & $\mathrm{~T}$ & $\mathrm{~F}$ & 41 & 7670000 \\
\hline ゆるやか & $\mathrm{F}$ & 0 & $\mathrm{~T}$ & $\mathrm{~T}$ & $\mathrm{~F}$ & 2 & 4440000 \\
\hline ライギョ & $\mathrm{T}$ & 0 & $\mathrm{~F}$ & $\mathrm{~F}$ & $\mathrm{~T}$ & 2 & 507000 \\
\hline 雷魚 & F & 2 & $\mathrm{~F}$ & $\mathrm{~T}$ & $\mathrm{~T}$ & 2 & 1030000 \\
\hline ラカディヴ & $\mathrm{T}$ & 0 & $\mathrm{~F}$ & $\mathrm{~F}$ & $\mathrm{~F}$ & 1 & 26 \\
\hline ラカディーヴ & $\mathrm{T}$ & 0 & $\mathrm{~F}$ & F & $\mathrm{F}$ & 1 & 6 \\
\hline レプトセファルス & $\mathrm{T}$ & 0 & $\mathrm{~F}$ & $\mathrm{~T}$ & $\mathrm{~T}$ & 5 & 11900 \\
\hline レプトケファルス & $\mathrm{T}$ & 0 & $\mathrm{~F}$ & $\mathrm{~F}$ & $\mathrm{~T}$ & 7 & 33400 \\
\hline レプトケパルス & $\mathrm{T}$ & 0 & $\mathrm{~F}$ & $\mathrm{~F}$ & $\mathrm{~T}$ & 4 & 771 \\
\hline ロードハウ & $\mathrm{T}$ & 0 & $\mathrm{~F}$ & $\mathrm{~F}$ & $\mathrm{~F}$ & 8 & 70500 \\
\hline ロードホウ & $\mathrm{T}$ & 0 & $\mathrm{~F}$ & $\mathrm{~F}$ & $\mathrm{~F}$ & 1 & 32 \\
\hline ロイヤルティ & $\mathrm{T}$ & 0 & $\mathrm{~F}$ & $\mathrm{~F}$ & $\mathrm{~T}$ & 1 & 4870000 \\
\hline ロヤルティ & $\mathrm{T}$ & 0 & $\mathrm{~F}$ & $\mathrm{~F}$ & $\mathrm{~F}$ & 1 & 385 \\
\hline
\end{tabular}

\subsection{Keyword Categories}

Since keywords should help to identify information, some words are unsuitable as keywords. One group of unsuitable words is the group of nouns in common use, and the other group is words appropriate in other articles but unsuitable in the WEB Fish Encyclopedia. Examples of the group of common nouns are "thing", "body", "object", "sake", "diver", and "fin", and examples of words unsuitable in the WEB Fish Encyclopedia are "Ministry of the Environment", "animal", "liver", "distribution", "pebbles", and "muscle". We obtained 4,946 keywords by sorting these nouns. We created seven categories to reveal the types of selected keywords. Table 3 shows the results. We used "General term of fish" and "Distribution" as rule (1) "General term or proper noun." 
Table 3. Keyword categories

\begin{tabular}{|c|c|c|c|}
\hline & $\begin{array}{l}\text { Examples } \\
\text { in Japanese }\end{array}$ & $\begin{array}{l}\text { Examples } \\
\text { in English }\end{array}$ & Total \\
\hline $\begin{array}{l}\text { General term of } \\
\text { fish }\end{array}$ & $\begin{array}{l}\text { スズキ } \\
\text { ハゼ } \\
\text { カサゴ }\end{array}$ & $\begin{array}{c}\text { Perch } \\
\text { Goby } \\
\text { Scorpionfish }\end{array}$ & 3983 \\
\hline Distribution & $\begin{array}{l}\text { 日本 } \\
\text { インド } \\
\text { 沖縄 }\end{array}$ & $\begin{array}{c}\text { Japan } \\
\text { India } \\
\text { Okinawa }\end{array}$ & 613 \\
\hline Living place & $\begin{array}{l}\text { 大陸棚 } \\
\text { 河口 } \\
\text { 深海 }\end{array}$ & $\begin{array}{c}\text { Continental shelf } \\
\text { Estuary } \\
\text { Deep sea }\end{array}$ & 80 \\
\hline Feature & $\begin{array}{l}\text { 軟体動物 } \\
\text { 毒性 } \\
\text { 斑点 }\end{array}$ & $\begin{array}{l}\text { Mollusk } \\
\text { Toxicity } \\
\text { Spot }\end{array}$ & 57 \\
\hline Body color & $\begin{array}{l}\text { 紫色 } \\
\text { 茶色 } \\
\text { ウグイス色 }\end{array}$ & $\begin{array}{c}\text { Violet } \\
\text { Brown } \\
\text { Greenish-brown }\end{array}$ & 33 \\
\hline $\begin{array}{l}\text { Part of general } \\
\text { term of fish }\end{array}$ & $\begin{array}{l}\text { ヨウジ } \\
\text { レッド } \\
\text { ボウ }\end{array}$ & $\begin{array}{l}\text { Pipe } \\
\text { Red } \\
\text { Robin }\end{array}$ & 93 \\
\hline Others & $\begin{array}{c}\text { 南方 } \\
\text { デトリタス } \\
\text { 模椂 }\end{array}$ & $\begin{array}{l}\text { South } \\
\text { Detritus } \\
\text { Pattern }\end{array}$ & 87 \\
\hline
\end{tabular}

\section{FURTHER DISCUSSION}

\subsection{Highlighted Display}

We used the obtained representative nouns for a highlighted display of documents. For example, we can highlight nouns in the WEB Fish Encyclopedia files by tagging them in HTML format, as shown in Figure 3. We put together all the representative nouns in a file and mapped them one by one to each of the WEB Fish Encyclopedia files. We were able to distinguish the types of keywords by tags such as <place 
name $>$ Kamchatka</place name> and to characterize the keywords individually. This procedure makes it easier to link to external open dictionaries such as Wikipedia.

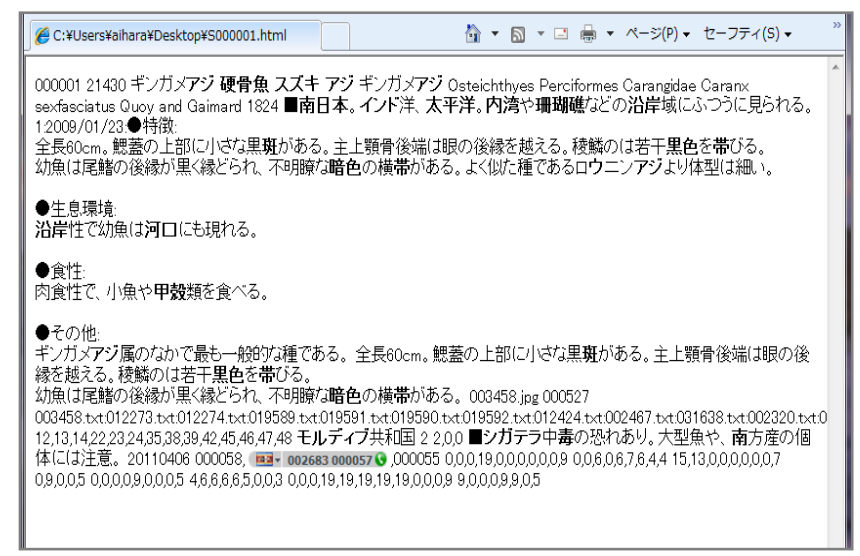

Figure 3. Example of tagging in a document

\subsection{Necessity for a Personal Dictionary}

We counted the number of keywords contained in the Japanese dictionary Kojien fourth edition and Wikipedia. The Japanese dictionary Kojien fourth edition contains only frequently used proper nouns. Also, the names of broad categories of fish were found. Furthermore, common nouns, such as nouns of habitats and features, were found. In Wikipedia, many proper nouns were found, but not many common nouns. According to the result of these observations, the Japanese dictionary Kojien fourth edition had 949 keywords out of 4,946, and Wikipedia had 1,238 keywords. As expected, the existing dictionaries did not cover all of the technical keywords, so we were able to prove the validity of making personal dictionaries.

\section{ACKNOWLEDGMENT}

The 4,668 text files of the WEB Fish Encyclopedia were provided by Mr. Kenichi Naoe, who is a student of the Graduate School of Information Science and Electrical Engineering, Kyushu University, and also a member of the Fishing Forum. Also, Prof. Masayoshi Aritsugi and Associate Prof. Teruaki Kitasuka of the Graduate School of Science and Technology, Kumamoto University gave valuable advice for this study.

\section{REFERENCES}

[1] I. Shinmura, Kojien fourth edition (Japanese Dictionary). Tokyo: 
Iwanami, 1991.

[2] Wikimedia Foundation, Inc. Wikipedia. Retrieved on January 31, 2012, from http://ja.wikipedia.org/.

[3] Fishing-Forum, The WEB fish encyclopedia (in Japanese, Sakanazukan). Retrieved on January 31, 2012, from http://www.fishing-forum.org/zukan/.

[4] D. Kawahara, and S. Kurohashi, Case frame compilation from the web using high-performance computing. In Nicoletta Calzolari (Ed.), Proceedings of the 5th International conference on Language Resource and Evaluation (p1344-1347). Italy: LREC 2006 Committees, 2006.

[5] S. Ravi, and K. Knight, Minimized models for unsupervised part-of-speech tagging. In Jian $\mathrm{Su}$ and Janyce Wiebe (Eds.), Proceedings of the Joint Conference of the 47th Annual Meeting of the $A C L$ and the 4th International Joint Conference on Natural Language (p504-512). Singapore: Curran Associates, 2009.http://dx.doi.org/10.3115/1687878.1687950.

[6] Z. Huang, V. Eidelman, and M. Harper, Improving a simple bigram hmm part-of-speech tagger by latent annotation and self-Training. In Mari Ostendorf (Ed.), Proceedings of Human Language Technologies: The 2009 Annual Conference of the North American Chapter of the Association for Computational Linguistics (p213-216). Stroudsburg: Association for Computational Linguistics, 2009.http://dx.doi.org/10.3115/1620853.1620911.

[7] J. Lafferty, A. McCallum, and F. Pereira, Conditional Random Fields: Probabilistic Models for Segmenting and Labeling Sequence Data, In Carla E. Brodley and Andrea Pohoreckyj Danyluk (Eds.), Proceeding of the 18th International Conference on Machine Learning (p282-289). San Francisco: Morgan Kaufmann, 2001.

[8] J. Halpern, Lexicon-based Orthographic Disambiguation in CJK Intelligent Information Retrieval. In Nicoletta Calzolari, Key-Sun Choi, Asanee Kawtrakul, Alessandro Lenci, and Tokunaga Takenobu (Eds.), Proceedings of the 3rd workshop on Asian language resources and international standardization (p1-7). Stroudsburg: Association for Computational Linguistics, 2002. http://dx.doi.org/10.3115/1118759.1118765. 